Research Paper

\title{
Cirrhosis in Wilson Disease is characterized by Impaired Hepatic Synthesis, Leukopenia and Thrombocytopenia
}

\author{
Hao-Jie Zhong1,2匹* , Ping Xiao ${ }^{2 *}$, Da Lin ${ }^{3^{*}}$, Hui-Min Zhou ${ }^{2}$, Xing-Xiang He ${ }^{2 \llbracket}$ \\ 1. Guangdong Medical University, Zhanjiang, China. \\ 2. Department of Gastroenterology, The First Affiliated Hospital of Guangdong Pharmaceutical University, Guangzhou, China. \\ 3. Department of Gastroenterology, Jieyang People's Hospital, Jieyang, China. \\ *These authors contributed equally to this work.
}

$\square$ Corresponding authors: Hao-Jie Zhong. Guangdong Medical University, No. 2 Wenmingdong Road, Zhanjiang, China, E-mail address: jaxzhong@126.com; Xing-Xiang He. Department of Gastroenterology, The First Affiliated Hospital of Guangdong Pharmaceutical University; No.19 Nonglinxia Road, Yuexiu District, Guangzhou, Guangdong, China, E-mail address: hexingxiang@gdpu.edu.cn.

( ) The author(s). This is an open access article distributed under the terms of the Creative Commons Attribution License (https://creativecommons.org/licenses/by/4.0/). See http:/ /ivyspring.com/terms for full terms and conditions.

Received: 2020.01.28; Accepted: 2020.05.21; Published: 2020.05.29

\begin{abstract}
Background: Patients with Wilson disease (WD) progress to cirrhosis at an early age but have good prognoses. This study aimed to delineate hepatic features in WD patients with or without cirrhosis.

Methods: Medical data were retrospectively collected from 27 July 2015 to 27 June 2018. WD patients were divided into two groups based on whether or not they progressed to cirrhosis. Liver function, portal hypertension features and hematocytopenia rates were compared between groups.

Results: The study enrolled 119 WD patients with cirrhosis and 53 WD patients without cirrhosis. There were no differences between groups for liver enzyme levels or incidence rates of Kayser-Fleischer ring (all $P>0.05$ ). Ascites and hepatic encephalopathy were nearly absent in both groups, and almost all patients were Child-Pugh group A. However, WD-associated cirrhotic patients had a higher prothrombin time (beta $=0.908, P=0.004$ ) and international normalized ratio (beta $=0.089, P=0.040$ ), wider portal vein diameter (beta $=1.330, P<0.001$ ), and an increased risk of splenomegaly/splenectomy (odds ratio $[\mathrm{OR}]=4.36,95 \%$ confidence interval $[\mathrm{Cl}]: 2.15-8.84, P<0.001)$. Moreover, WD-associated cirrhotic patients have significantly increased risks of leukopenia $(\mathrm{OR}=2.30,95 \% \mathrm{Cl}: 1.00-5.25, P=0.049)$ and thrombocytopenia (OR = 6.89, 95\% Cl: 2.01-23.59, $P=0.002)$.

Conclusions: Despite presenting good outcomes, mild hepatocyte injury, and good hepatic metabolic function, WD-associated cirrhotic patients show more serious impairment of hepatic synthetic function, wider portal vein diameter, and higher risk of splenomegaly due to portal hypertension.
\end{abstract}

Key words: Wilson disease; Cirrhosis; Liver dysfunction; Portal hypertension; Splenomegaly

\section{Introduction}

Cirrhosis is the eleventh-most common cause of death globally and is characterized by hepatic dysfunction, portal hypertension, and high risk of hepatocellular carcinoma (HCC) [1,2]. Compared with those with liver disease and no cirrhosis, cirrhotic patients often showed more severe hepatocyte injury; worse declines in hepatic synthetic function; and higher prevalence rates of variceal bleeding, encephalopathy, and splenomegaly, especially among cases caused by non-alcoholic fatty liver disease and viral hepatitis [3-5]. It is these manifestations and complications, rather than the presence of comorbidities, which should account for the increased mortality of cirrhotic patients $[1,6]$. Therefore, it is of great importance to clarify the hepatic features in patients with or without cirrhosis.

Wilson disease (WD) is an autosomal recessive disorder of copper metabolism, caused by mutations 
in the $A T P 7 B$ gene that encodes a copper-transporting P-type ATPase [7]. Unlike most cases of cirrhosis attributable to other causes, patients with WD frequently progress to cirrhosis at a young age, one-third while still children or adolescents $[8,9]$. Nevertheless, the prognosis of WD-associated cirrhosis is excellent, and appropriate treatment can normalize many laboratory test results [10]. Longterm follow-up studies found no increased risk of HCC $(0.14 \%$ /year $)$ in WD-associated cirrhotic patients $[11,12]$. Interestingly, the hepatic features of WD-associated cirrhosis are very distinct from those of hepatitis B-associated cirrhosis. Patients present better liver function but a higher risk of splenomegaly, suggesting that cirrhosis is not a single disease entity [13]. Therefore, it is crucial to characterize the hepatic features in WD patients with or without cirrhosis to guide further treatment and prevent complications. Thus, we performed this study to delineate the hepatic features in WD patients with or without cirrhosis.

\section{Methods}

\section{Study design and participants}

This cross-sectional study was performed at the First Affiliated Hospital of Guangdong Pharmaceutical University, Guangzhou, China. The subjects were inpatients who had been diagnosed with WD at this hospital from 27 July 2015 to 27 June 2018. The patients were excluded if they met any of the following criteria: (1) carcinoma; (2) severe heart or pulmonary diseases; (3) other hepatic conditions such as alcoholic, fatty, viral, autoimmune, druginduced, or parasitic liver disease; or (4) important medical data missing. Each patient was recorded only once.

This study was performed with the approval of the First Affiliated hospital of Guangdong Pharmaceutical University Institutional Review Board (No. 2019-60) and adhered to the Declaration of Helsinki. Given that this was a retrospective study, the Institutional Review Board waived the requirement for informed consent from each patient.

\section{Data collection}

We reviewed medical data of all enrolled subjects to collect the information on gender, age at time of data collection, body mass index (BMI); history of alcohol consumption and smoking; underlying diseases (diabetes and hypertension); duration and manifestations of WD; imaging findings; and laboratory test results including alanine transaminase (ALT), aspartate transaminase (AST), serum albumin, prothrombin time (PT), international normalized ratio (INR), total cholesterol, triglyceride, bilirubin, white blood cell (WBC) count, red blood cell (RBC) count, and platelet count.

Alcohol use was defined as the alcohol consumption $>140 \mathrm{~g}$ per week. Hypoalbuminemia was defined as serum albumin $<30 \mathrm{~g} / \mathrm{L}$. Hyperbilirubinemia was defined as bilirubin $>34$ $\mu \mathrm{mol} / \mathrm{L}$. Leukopenia was diagnosed in patients with WBC counts $<4.0 \times 10^{9} /$ L. Erythropenia was defined as $\mathrm{RBC}$ count $<4.0 \times 10^{12} / \mathrm{L}$ (male) or $<3.5 \times 10^{12} / \mathrm{L}$ (female). Thrombocytopenia was diagnosed at platelet counts $<100 \times 10^{9} / \mathrm{L}$. In China, cirrhosis was diagnosed based on liver biopsy or combination of clinical manifestations (hepatomegaly, splenomegaly, ascites, hepatic encephalopathy and others), laboratory examinations (ALT, AST, procollagen type III N-terminal propeptide, type IV collagen, laminin, hyaluronic acid and others), and imaging examinations (typical morphological changes, including a blunted, nodular liver edge accompanied by splenomegaly in abdominal ultrasonography or computed tomography).

\section{Clinical diagnosis of WD}

In China, WD is diagnosed based on a combination of clinical features and laboratory parameters: onset age, family history, symptoms, serum ceruloplasmin $<200 \mathrm{mg} / \mathrm{L}$, urinary copper excretion $\geq 100 \mu \mathrm{g} / 24 \mathrm{~h}$; liver copper $>250 \mu \mathrm{g} / \mathrm{g}$ dry weight; urinary copper excretion $>1600 \mu \mathrm{g} / 24 \mathrm{~h}$ after the administration of $2 \times 500 \mathrm{mg}$ D-penicillamine; presence of Kayser-Fleischer ring by slit-lamp examination; and mutation analysis [14]. Although the Chinese diagnosis criteria were not based on a score system, all the tests in the international criteria were included in these criteria [15].

\section{Statistical analysis}

Statistical analyses were performed using SPSS software, version 22 (IBM Corp., Armonk, NY, USA). Results are presented as frequencies and proportions for categorical variables, means \pm standard deviation for normally distributed continuous variables, and medians and interquartile ranges for non-normally distributed continuous variables. Chi-square or Fisher exact tests were used for group comparisons of categorical variables. Student $t$-tests were performed for group comparisons of normally distributed data, and Mann-Whitney $U$-tests were applied for nonnormally distributed data. To assess the association between cirrhosis and laboratory results in WD patients, multivariable linear regression analysis with a stepwise method was used to adjust for confounders (i.e. age, sex, body mass index, disease duration, hypertension, diabetes, alcohol use, and smoking). Logistic regression analysis with a backward 
conditional method was carried out to assess the relationship of hepatic features with cirrhosis. The results are reported as odds ratios (ORs) and 95\% confidence intervals (CIs). Two-sided $P$-values $<0.05$ were considered statistically significant.

Table 1. Patient characteristics

\begin{tabular}{llll}
\hline & Cirrhosis $(\mathrm{n}=119)$ & Non-cirrhosis $(\mathrm{n}=53)$ & $P$-value \\
\hline Age $(\mathrm{yr})$ & $25.00(21.00-33.00)$ & $21.00(14.50-29.00)$ & 0.003 \\
Male sex & $56(47.06)$ & $29(54.72)$ & 0.354 \\
Body mass index $\left(\mathrm{kg} / \mathrm{m}^{2}\right)$ & $19.61(17.98-21.78)$ & $18.85(16.72-20.64)$ & 0.016 \\
Disease duration (years) & $(\mathrm{n}=119)$ & $(\mathrm{n}=52)$ & 0.971 \\
$<10$ & $77(64.71)$ & $33(63.46)$ & \\
$10-19$ & $34(28.57)$ & $15(28.85)$ & \\
$\geq 20$ & $8(6.72)$ & $4(7.69)$ & 1.000 \\
Diabetes & $1(0.84)$ & $0(0)$ & 1.000 \\
Hypertension & $1(0.84)$ & $0(0)$ & 0.523 \\
Alcoholism & $1(0.84)$ & $1(1.89)$ & 0.511 \\
Smoking & $12(10.08)$ & $3(5.66)$ & 0.009 \\
Neurological & $85(71.43)$ & $27(50.94)$ & \\
manifestations & & $0(0)$ & 0.179 \\
Psychiatric manifestations & $6(5.04)$ &
\end{tabular}

\section{Results}

\section{Patient characteristics}

After excluding six patients with carcinoma and seven patients with severe heart or pulmonary diseases, 119 WD patients with cirrhosis and 53 WD patients without cirrhosis were enrolled. The clinical characteristics of the two groups are presented in Table 1. Most of the diagnoses of cirrhosis (103/119) were made based on patients' medical history. Sixteen patients were newly diagnosed cirrhosis. All enrolled patients were undergoing copper lowering treatment for WD.

\section{Liver function features in WD patients with or without cirrhosis}

WD patients with cirrhosis had significantly lower levels of albumin and triglyceride, while PT, INR, and bilirubin level were significantly higher compared to WD patients without cirrhosis $(P<0.05$, Table 2). The total cholesterol level was higher in WD-associated cirrhotic patients, but the difference was not significant (Table 2). There were no significant between-group differences for ALT and AST levels, and incidence rates of Kayser-Fleischer ring. Hepatic encephalopathy was almost absent in both groups, and almost all patients were in ChildPugh group A.

After adjustment for confounders, WDassociated cirrhotic patients still showed higher PT and INR and lower levels of total cholesterol and triglyceride; there were no differences for other liver function indices compared with patients with WD without cirrhosis (Tables 3 and 4).

Table 2. Hepatic features in WD patients with or without cirrhosis

\begin{tabular}{|c|c|c|c|}
\hline & Cirrhosis $(n=119)$ & Non-cirrhosis $(n=53)$ & $P$-value \\
\hline \multicolumn{4}{|l|}{ Liver function features } \\
\hline $\operatorname{ALT}(\mathrm{U} / \mathrm{L})$ & $22.00(15.00-34.00)$ & $23.00(14.00-55.50)$ & 0.508 \\
\hline AST (U/L) & $23.00(18.00-38.00)$ & $23.00(18.00-38.90)$ & 0.797 \\
\hline Albumin (g/L) & $39.00(36.00-41.10)$ & $40.00(36.65-44.00)$ & 0.044 \\
\hline PT (s) & $14.40(13.60-15.30)$ & $13.50(12.95-14.25)$ & $<0.001$ \\
\hline INR & $1.12(1.04-1.21)$ & $1.03(0.97-1.10)$ & $<0.001$ \\
\hline Total cholesterol (mmol/L) & $3.67(3.06-4.45)(n=88)$ & $3.98(3.65-4.66)(n=40)$ & 0.054 \\
\hline Triglyceride (mmol/L) & $0.82(0.62-1.06)(\mathrm{n}=88)$ & $0.98(0.81-1.32)(n=40)$ & 0.010 \\
\hline Bilirubin ( $\mu \mathrm{mol} / \mathrm{L})$ & $13.30(9.10-18.10)$ & $11.10(7.75-13.75)$ & 0.010 \\
\hline Hepatic encephalopathy & $1(0.84)$ & $0(0)$ & 1.000 \\
\hline Kayser-Fleischer ring & $39(32.77)$ & $13(24.53)$ & 0.277 \\
\hline Child-Pugh classification & & & 0.225 \\
\hline A & $113(94.96)$ & $53(100)$ & \\
\hline $\mathrm{B} / \mathrm{C}$ & $6(5.04)$ & $0(0)$ & \\
\hline \multicolumn{4}{|l|}{ Portal hypertension features } \\
\hline Portal vein diameter (mm) & $10.00(10.00-11.00)(n=88)$ & $9.00(8.00-10.00)(n=40)$ & $<0.001$ \\
\hline Ascites & $3(2.52)$ & $1(1.89)$ & 1.000 \\
\hline Splenomegaly/splenectomy & $76(63.87)$ & $15(28.30)$ & $<0.001$ \\
\hline \multicolumn{4}{|l|}{ Hematocytopenia } \\
\hline WBC $\left(10^{9} / \mathrm{L}\right)$ & $4.60(3.38-5.75)$ & $5.04(4.43-6.50)$ & 0.012 \\
\hline $\operatorname{RBC}\left(10^{12} / \mathrm{L}\right)$ & $4.37(3.93-4.83)$ & $4.61(4.14-4.96)$ & 0.021 \\
\hline Platelet $\left(10^{9} / \mathrm{L}\right)$ & $170.96 \pm 89.59$ & $227.49 \pm 93.00$ & $<0.001$ \\
\hline Leukopenia & $39(33.05)$ & $9(16.98)$ & 0.031 \\
\hline Erythropenia & $16(13.56)$ & $5(9.43)$ & 0.447 \\
\hline Thrombocytopenia & $35(29.66)$ & $3(5.66)$ & $<0.001$ \\
\hline
\end{tabular}

Data are presented as means \pm standard deviation, medians (interquartile ranges) or $\mathrm{n}(\%)$. ALT, alanine transaminase; AST, aspartate transaminase; INR, international normalized ration; PT, prothrombin time; RBC, red blood cell; WBC, white blood cell; WD, Wilson disease. 
Table 3. Multivariable linear regression analysis of hepatic features in WD patients with and without cirrhosis

\begin{tabular}{llll}
\hline & Beta & Standard error & $P$-value \\
\hline Liver function indices & & & \\
ALT $(\mathrm{U} / \mathrm{L})$ & - & - & - \\
AST $(\mathrm{U} / \mathrm{L})$ & - & - & - \\
Albumin $(\mathrm{g} / \mathrm{L})$ & - & - & - \\
PT $(\mathrm{s})$ & 0.908 & 0.314 & 0.004 \\
INR & 0.089 & 0.032 & 0.040 \\
Total cholesterol (mmol/L) & -0.400 & 0.199 & 0.047 \\
Triglyceride $(\mathrm{mmol} / \mathrm{L})$ & -0.299 & 0.145 & 0.041 \\
Bilirubin $(\mu \mathrm{mol} / \mathrm{L})$ & - & - & - \\
Portal hypertension & & & $<0.001$ \\
Portal vein diameter $(\mathrm{mm})$ & 1.330 & 0.320 & \\
Hematocytopenia & & & 0.014 \\
WBC $\left(10^{9} / \mathrm{L}\right)$ & -0.709 & 0.286 & 0.048 \\
RBC $\left(10^{12} / \mathrm{L}\right)$ & -0.253 & 0.127 & $<0.001$ \\
Platelet $\left(10^{9} / \mathrm{L}\right)$ & -71.016 & 13.615 & \\
\hline
\end{tabular}

Adjusted for age, sex, body mass index, disease duration, hypertension, diabetes, alcoholism and smoking. ALT, alanine transaminase; AST, aspartate transaminase; INR, international normalized ration; PT, prothrombin time; RBC, red blood cell; WBC, white blood cell; WD, Wilson disease.

Table 4. Logistic regression analysis of hepatic features in WD patients with and without cirrhosis

\begin{tabular}{llll}
\hline & OR & $95 \% \mathrm{CI}$ & $P$-value \\
\hline Liver function indices & & & - \\
Hepatic encephalopathy & - & - & - \\
Kayser-Fleischer ring & - & - & - \\
$\begin{array}{l}\text { Child-Pugh classification } \\
\text { Portal hypertension }\end{array}$ & - & - & \\
$\begin{array}{l}\text { Ascites } \\
\text { Splenomegaly/splenectomy }\end{array}$ & - & & - \\
$\begin{array}{l}\text { Hematocytopenia } \\
\text { Leukopenia }\end{array}$ & 2.36 & $2.15-8.84$ & $<0.001$ \\
Erythropenia & - & $1.00-5.25$ & 0.049 \\
Thrombocytopenia & 6.89 & - & - \\
\hline
\end{tabular}

Adjusted for age, sex, body mass index, disease duration, hypertension, diabetes, alcoholism and smoking. CI, confidence interval; OR, odds ratio; WD, Wilson disease.

\section{Portal hypertension features in WD patients with or without cirrhosis}

The portal vein diameter and prevalence of splenomegaly (or splenectomy) were significantly higher in WD-associated cirrhotic patients (Table 2). And ascites was almost absent in both groups.

Multivariable linear regression after confounder adjustment showed that WD-associated cirrhotic patients had a wider portal vein diameter (beta $=$ 1.330, $P<0.001$, Table 3). Moreover, logistic regression analysis showed that WD-associated cirrhotic patients also had a significantly increased risk of splenomegaly (or splenectomy) compared with non-cirrhotic patients (OR $=4.36,95 \%$ CI: 2.15-8.84, $P$ $<0.001$, Table 4).

\section{Hematocytopenia in WD patients with or without cirrhosis}

Compared with those without cirrhosis, WBC, $\mathrm{RBC}$ and platelet counts were significantly decreased in WD-associated cirrhotic patients, both before and after adjustment for confounders (Tables 2 and 3). The incidence of leukopenia $(33.05 \%$ vs. $16.98 \%, P=$ $0.031)$ and thrombocytopenia $(29.66 \%$ vs. $5.66 \%, P<$ 0.001 ) were also higher in WD patients with cirrhosis (Table 2). After adjustment, patients with WDassociated cirrhosis showed significantly increased risks of leukopenia $(\mathrm{OR}=2.30,95 \% \mathrm{CI}$ : 1.00-5.25, $P=$ $0.049)$ and thrombocytopenia $(\mathrm{OR}=6.89,95 \% \mathrm{CI}$ : 2.01-23.59, $P=0.002$; Table 4).

Seventy-six patients with WD-associated cirrhosis had splenomegaly; 8 underwent partial splenectomy, 12 had complete splenectomy, and 56 did not undergo splenectomy (included 1 patient with no routine blood test results). Only $8.33 \%(1 / 12)$ patients with complete splenectomy had leukopenia and $0 \%(0 / 12)$ had thrombocytopenia, which were much lower rates compared to patients who underwent partial splenectomy $(87.50 \%$ and $62.50 \%)$ or did not have surgery ( $45.45 \%$ and $45.45 \%$, Table 5 ).

\section{Discussion}

Cirrhosis, as an end-stage liver disease, leads to liver dysfunction and portal hypertension that can cause fatal complications such as liver failure, variceal bleeding, hepatic encephalopathy, severe infections, and even death $[2,16]$. Mounting evidence suggests that cirrhosis should no longer be considered a single disease, because patients with cirrhosis due to different causes may present with variable clinical features [2,13]. WD patients progress to cirrhosis at an early age but have good prognosis, which might contribute to its distinct hepatic features. However, the clinical features that distinguish WD patients with and without cirrhosis are still unclear. To the best of our knowledge, this is the first study to compare liver features in these two groups of WD patients. The findings might help facilitate early diagnosis and prompt treatment to prevent complications when WD patients progress to cirrhosis.

Table 5. Splenectomy for WD-associated cirrhotic patients with splenomegaly

\begin{tabular}{lllll}
\hline & No splenectomy $(\mathrm{n}=55)$ & Partial splenectomy $(\mathrm{n}=8)$ & Complete splenectomy $(\mathrm{n}=12)$ & P-value \\
\hline Leukopenia & $25(45.45)$ & $7(87.50)$ & $1(8.33)$ & 0.002 \\
Erythropenia & $9(16.36)$ & $3(37.50)$ & $3(25.00)$ & 0.337 \\
Thrombocytopenia & $25(45.45)$ & $5(62.50)$ & $0(0)$ & 0.006 \\
\hline
\end{tabular}

Data are presented as $\mathrm{n}(\%)$. WD, Wilson disease. 
As a comprehensive index to evaluate liver functional reserve, the Child-Pugh classification is widely used to predict the prognosis of cirrhotic patients [17]. We found that almost all WD-cirrhosis patients were in Child-Pugh group A, which predicted good outcomes in WD-associated cirrhosis patients, similar with previous studies $[7,10]$. Cirrhotic patients typically present more with serious hepatocyte injury compared with non-cirrhotic patients $[4,18]$. However, we found no difference in hepatocyte injury (based on ALT and AST) between WD patients with and without cirrhosis. Consistent with this, previous studies showed that aminotransferase levels could decrease and reach nearly normal values in WD patients who undergone copper lowering treatment, despite the continued presence of cirrhosis $[19,20]$. A long-term follow-up study assessed liver fibrosis in WD patients by biopsy and found marked reductions of liver fibrosis in WD-associated cirrhotic patients on copper removal treatment [21]. Their results demonstrated that advanced fibrosis could reverse in controlled WD. This suggests that the prognosis for WD-associated cirrhotic patients might improve with appropriate treatment.

The liver plays a crucial role in synthesizing albumin, coagulation factors, and lipids and is involved in ammonia and bilirubin metabolism [22]. As a feature of cirrhosis, liver dysfunction was assessed based on the synthesis and metabolism of these substances. The incidences of hypoalbuminemia $(5.04 \%)$, hyperbilirubinemia $(4.20 \%)$, and hepatic encephalopathy $(0.84 \%)$ were low in WD-associated cirrhotic patients. Moreover, multivariable linear and logistic regression analyses showed no differences in albumin level, bilirubin level between the two groups. These findings suggest that WD patients seem to have good liver metabolic functions despite the presence of cirrhosis. However, compared with those without cirrhosis, WD patients with cirrhosis presented higher PT and INR and lower total cholesterol and triglyceride levels, which indicated they have impaired synthesis of coagulation factors and lipids.

As another feature of cirrhosis, portal hypertension was assessed based on the presence of ascites, portal vein diameter, and splenomegaly. Ascites was almost absent in both groups, and cirrhosis was not associated with a higher risk of ascites in WD patients. This might be due to the high concentration of serum albumin in cirrhotic patients, which might result in high plasma osmotic pressure and reduce the occurrence of ascites [23]. Nevertheless, compared with those without cirrhosis, WD-associated cirrhotic patients had a wider portal vein diameter. This indicates that these patients might have higher portal vein pressure that could predispose them to esophageal varices [24].

In normal physiology, splenic blood flows back to the liver through the portal vein. In cirrhosis, spleen congestion secondary to portal outflow resistance leads to splenomegaly, which is an important clinical indicator of portal hypertension [25]. A previous report described the clinical features of 910 cirrhotic patients showed that about half $(50.50 \%)$ presented with splenomegaly [26]. The present study found that the incidence of splenomegaly in WD-associated cirrhotic patients was higher than that in non-cirrhotic patients $(63.87 \%$ vs. $28.30 \%)$ and also higher than in the previous study $(50.50 \%)$ [26]. After adjustment for potential confounders, WD-associated cirrhotic patients showed a 4.36-fold risk of splenomegaly compared to non-cirrhotic patients.

Splenomegaly in WD patients frequently causes functional overactivity of the spleen (known as hypersplenism), and some anti-copper medications such as penicillamine can occasionally cause bone marrow depression, anemia, and leukopenia [27,28]. Therefore, we also assessed hematocytopenia rates in both groups [29]. We found that patients with WD-associated cirrhosis showed reduced WBC, RBC, and platelet counts and increased risks of leukopenia and thrombocytopenia, which might increase their risks of serious infection and spontaneous bleeding [27]. A previous study demonstrated that infections increase mortality by four-fold in patients with cirrhosis; $30 \%$ die within a month after infection and another $30 \%$ die by 1 year, and variceal bleeding and intracerebral bleeding due to thrombocytopenia can also be fatal [30]. Thus, we should closely evaluate WD patients for leukopenia and thrombocytopenia when they progress to cirrhosis.

A study from China included 65 patients with splenomegaly secondary to cirrhosis and showed improvements in WBC and platelet counts after splenectomy [31]. Additionally, another study of 70 WD patients with splenomegaly reported a similar result [27]. Consistent with these results, we found that the incidence rates of leukopenia and thrombocytopenia were significantly reduced in WD patients who underwent complete splenectomy to treat splenomegaly secondary to cirrhosis. This finding revealed that complete splenectomy was a useful treatment for leukopenia and thrombocytopenia in WD-associated cirrhotic patients, and it was more efficient than partial splenectomy.

There are several limitations of this study. First, there was the possibility of selection bias, since we only enrolled in-patients who might have more severe 
WD and more comorbid diseases compared with outpatients. Second, some hepatic complications such as esophageal varices, hepatorenal syndrome, and hepatocellular carcinoma were hard to assess due to a lack of clinical data. Third, to explore the effect of splenectomy on treatment of hematocytopenia, to compare the data of patients before and after splenectomy was the most ideal way. However, due to the lack of data before splenectomy, we only compared the data among with complete splenectomy, partial splenectomy and nonsplenectomy. Fourth, although WD is rare, the sample size of our study was still small. These results should be interpreted with caution, and further confirmatory studies are needed.

\section{Conclusions}

In conclusion, despite good outcomes, mild hepatocyte injury, and good hepatic metabolic function, patients with WD-associated cirrhotic showed more serious impairment of hepatic synthetic function, wider portal vein diameter, and higher risk of splenomegaly due to portal hypertension. More attention should be paid to the insufficiency of coagulation factors due to hepatic synthetic dysfunction and leukopenia and thrombocytopenia due to hypersplenism; addressing these areas could lower the risks of infection and bleeding. Complete splenectomy might be an effective therapeutic measure for leukopenia and thrombocytopenia in WD-associated cirrhotic patients.

\section{Acknowledgements}

This study was supported by the National Nature Science Foundation of China (Grant No. 81602110).

\section{Competing Interests}

The authors have declared that no competing interest exists.

\section{References}

1. Asrani SK, Devarbhavi H, Eaton J, Kamath PS. Burden of liver diseases in the world. J Hepatol. 2019; 70: 151-71.

2. Tsochatzis EA, Bosch J, Burroughs AK. Liver cirrhosis. Lancet. 2014; 383: 1749-61.

3. Nishida T, Kobashi H, Fujioka S, Fujio K, Takaguchi K, Ikeda H, et al. A prospective and comparative cohort study on efficacy and drug resistance during long-term lamivudine treatment for various stages of chronic hepatitis B and cirrhosis. J Gastroenterol Hepatol. 2008; 23: 794-803.

4. Kessoku T, Ogawa Y, Yoneda M, Imajo K, Sumida Y, Eguchi Y, et al. Simple scoring system for predicting cirrhosis in nonalcoholic fatty liver disease. World J Gastroenterol 2014; 20: 10108-14.

5. Zeng DW, Zhang JM, Liu YR, Dong J, Wu YL, Lin S, et al. A new model for predicting liver cirrhosis in chronic hepatitis $\mathrm{B}$ virus carriers with low serum alanine transaminase activity. Clin Res Hepatol Gastroenterol. 2014; 38: 727-34.

6. D'Amico G, Morabito A, D'Amico M, Pasta L, Malizia G, Rebora P, et al. Clinical states of cirrhosis and competing risks. J Hepatol. 2018; 68: 563-76.

7. Czlonkowska A, Litwin T, Dusek P, Ferenci P, Lutsenko S, Medici V, et al. Wilson disease. Nat Rev Dis Primers. 2018; 4: 21
8. Ferenci P, Stremmel W, Czlonkowska A, Szalay F, Viveiros A, Stattermayer $\mathrm{AF}$, et al. Age and sex but not ATP7B genotype effectively influence the clinical phenotype of Wilson disease. Hepatology. 2019; 69: 1464-76.

9. Roberts EA, Socha P. Wilson disease in children. Handb Clin Neurol. 2017; 142: 141-56.

10. Boga S, Ala A, Schilsky ML. Hepatic features of Wilson disease. Handb Clin Neurol. 2017; 142: 91-9.

11. Gerosa C, Fanni D, Congiu T, Piras M, Cau F, Moi M, et al. Liver pathology in Wilson's disease: from copper overload to cirrhosis. J Inorg Biochem. 2019; 193: 106-11.

12. van Meer S, de Man RA, van den Berg AP, Houwen RH, Linn FH, van Oijen $\mathrm{MG}$, et al. No increased risk of hepatocellular carcinoma in cirrhosis due to Wilson disease during long-term follow-up. J Gastroenterol Hepatol. 2015; 30: 535-39.

13. Zhong HJ, Sun HH, Xue LF, McGowan EM, Chen Y. Differential hepatic features presenting in Wilson disease-associated cirrhosis and hepatitis B-associated cirrhosis. World J Gastroenterol. 2019; 25: 378-87.

14. Liang XL YR, Wu ZY, Wang N, Li XH, Wang X. Guideline of Wilson's disease diagnosis and treatment. Chin J Neurol. 2008; 41: 566-9.

15. Ferenci P, Caca K, Loudianos G, Mieli-Vergani G, Tanner S, Sternlieb I, et al. Diagnosis and phenotypic classification of Wilson disease. Liver Int. 2003; 23: $139-42$.

16. Hernaez R, Sola E, Moreau R, Gines P. Acute-on-chronic liver failure: an update. Gut. 2017; 66: 541-53

17. Peng Y, Qi X, Guo X. Child-pugh versus MELD score for the assessment of prognosis in liver cirrhosis: a systematic review and meta-analysis of observational studies. Medicine. 2016; 95: e2877.

18. Li Q, Lu C, Li W, Huang Y, Chen L. Evaluation of eLIFT for non-invasive assessment of liver fibrosis and cirrhosis in patients with chronic hepatitis B virus infection. Sci Rep. 2017; 7: 5429.

19. Schilsky ML, Scheinberg IH, Sternlieb I. Prognosis of Wilsonian chronic active hepatitis. Gastroenterology. 1991; 100: 762-7.

20. Liver EAfSo. EASL Clinical Practice Guidelines: Wilson's disease. J Hepatol. 2012; 56: 671-85.

21. Askari FK, Greenson J, Dick RD, Johnson VD, Brewer GJ. Treatment of Wilson's disease with zinc. XVIII. Initial treatment of the hepatic decompensation presentation with trientine and zinc. J Lab Clin Med. 2003; 142: 385-90.

22. Tsai MT, Tarng DC. Beyond a measure of liver function-bilirubin acts as a potential cardiovascular protector in chronic kidney disease patients. Int J Mol Sci. 2018; 20.

23. Piano S, Tonon M, Angeli P. Management of ascites and hepatorenal syndrome. Hepatol Int. 2018; 12: 122-34.

24. Bhattarai S, Dewan KR, Shrestha G, Patowary BS. Non-invasive predictors of gastro-oesophageal varices. JNMA J Nepal Med Assoc. 2017; 56: 298-303.

25. Udell JA, Wang CS, Tinmouth J, FitzGerald JM, Ayas NT, Simel DL, et al. Does this patient with liver disease have cirrhosis? JAMA. 2012; 307: 832-42.

26. Orlando R, Lirussi F, Basso SM, Lumachi F. Splenomegaly as risk factor of liver cirrhosis. A retrospective cohort study of 2,525 patients who underwent laparoscopy. In Vivo. 2011; 25: 1009-12.

27. Li LY, Yang WM, Chen HZ, Wu YH, Fang X, Zhang J, et al. Successful splenectomy for hypersplenism in Wilson's disease: a single center experience from China. PLoS One. 2015; 10: e0124569.

28. Dziezyc K, Litwin T, Czlonkowska A. Other organ involvement and clinical aspects of Wilson disease. Handb Clin Neurol. 2017; 142: 157-69.

29. Lv Y, Yee Lau W, Wu H, Han X, Gong X, Liu N, et al. Causes of peripheral cytopenia in hepatitic cirrhosis and portal hypertensive splenomegaly. Exp Biol Med (Maywood). 2017; 242: 744-9.

30. Arvaniti V, D'Amico G, Fede G, Manousou P, Tsochatzis E, Pleguezuelo M, et al. Infections in patients with cirrhosis increase mortality four-fold and should be used in determining prognosis. Gastroenterology. 2010; 139: 1246-1256, 56 e1-5.

31. Jiao S, Chen H, Wang Y, Zhu J, Tan J, Gao J. Splenectomy versus partial splenic embolization for massive splenomegaly secondary to hepatitis B-related liver cirrhosis: a case-control study. Gastroenterol Res Pract. 2016; 2016: 3471626. 